\title{
PROGNOSTIC FACTORS AND LONG-TERM RESULTS AFTER THYMOMA RESECTION: A SERIES OF 307 PATIENTS
}

Jean-François Regnard, $\mathrm{MD}^{\mathrm{a}}$

Pierre Magdeleinat, $\mathrm{MD}^{\mathrm{a}}$

Christian Dromer, $\mathrm{MD}^{\mathrm{b}}$

Elisabeth Dulmet, $\mathrm{MD}^{\mathrm{b}}$

Vincent De Montpreville, $\mathrm{MD}^{\mathrm{b}}$

Jean-François Levi, MD ${ }^{\mathrm{a}}$

Philippe Levasseur, $\mathrm{MD}^{\mathrm{a}}$
Three hundred seven cases of patients who underwent operation for thymoma (196 of whom had myasthenia gravis) were analyzed to assess the prognostic values of Masaoka clinical staging, completeness of resection, histologic classification, history of myasthenia gravis, and postoperative radiotherapy. According to the Masaoka staging system, 135 thymomas were stage I, 70 were stage II, 83 were stage III, and 19 were stage IV. According to the Verley and Hollmann histologic classification system, 67 thymomas were type 1,77 were type 2,139 were type 3 , and 24 were type 4 . Two hundred sixty patients underwent complete resection, 30 underwent incomplete resection, and 17 underwent biopsy. Postoperative radiotherapy was performed mainly in cases of invasive or metastatic thymoma. Mean follow-up was 8 years; eight patients were unavailable for follow-up. The overall 10- and 15-year survivals were $67 \%$ and $57 \%$, respectively. In univariate analysis, three prognostic factors were established: completeness of resection, Masaoka clinical staging, and histologic classification. Furthermore, among patients with stage III thymomas, survival was significantly higher for patients with complete resection than for patients with incomplete resection $(p<0.001)$. Completeness of resection should therefore be taken into account in clinical-pathologic staging. We did not find any significant difference with respect to disease-free survival between patients who had postoperative radiotherapy and those who did not. In multivariate analysis, the sole significant prognostic factor was completeness of resection. On the basis of these findings, a new clinical-pathologic staging system is proposed. (J Thorac Cardiovasc Surg 1996;112:376-84)
Thestis he prognostic factors for thymoma are difficult to establish and remain controversial. ${ }^{1-7}$ There are various reasons for this; these tumors are uncommon, they are clinically and anatomically polymorphic, they grow slowly, several histological classifications have been used, and the series published to date are all retrospective. The aim of this study was to evaluate the prognostic values of clinical-pathologic staging, completeness of resection, histologic

From the Departments of Thoracic and Vascular Surgery and Pathology, Marie Lannelongue Hospital, Le Plessis-Robinson, France.

Received for publication June 16, 1995; revisions requested Oct. 31, 1995; revisions received Jan. 16, 1996; accepted for publication Jan. 16, 1996.

Address for reprints: Jean-François Regnard, Hopital Marie Lannelongue, 133 Ave. de la Résistance, Le Plessis-Robinson, France.

Copyright @ 1996 by Mosby-Year Book, Inc.

$0022-5223 / 96 \$ 5.00+0 \quad \mathbf{1 2 / 1 / 7 1 9 1 9}$ classification, association with myasthenia gravis (MG), and use of postoperative radiotherapy.

\section{Patients and methods}

Patients. Thymoma has been defined as a tumor originating from the epithelial cells of the thymus. We retrospectively studied data from 307 consecutive patients operated on between 1955 and 1993 in the department of Thoracic and Vascular Surgery of the Marie Lannelongue Hospital. There were 180 female (59\%) and 127 male patients. The mean age was 49 years, ages ranged from 6 to 77 years.

One hundred ninety-five patients (64\%) had MG. Eighteen patients had another autoimmune disease (OAD), isolated (14) or associated with MG (four): thyroiditis (six), systemic lupus erythematous (four), pure red cell aplasia (four), hypogammaglobulemia (two), Sjögren's disease (two), pemphigus vulgaris (one), or hemolytic anemia (one). Ninety-seven patients had no autoimmune disease (NAD).

The autoimmune disease revealed the thymoma in $85 \%$ of patients with $\mathrm{MG}$ and $65 \%$ of patients with OAD patients. Systematic chest radiography and mediastinal 
compression symptoms revealed thymoma in two-thirds and one-third of patients with NAD, respectively.

Methods. We performed a retrospective study to determine long-term outcome after thymoma resection. Thymomas were classified according to the Masaoka clinicalpathologic staging system ${ }^{8}$ and to the Verley histologic classification system. ${ }^{9}$

At the beginning of our experience, patients with laterally located thymomas were operated on through a lateral thoracotomy; those with centrally located thymomas were operated on through a sternotomy or an anterolateral thoracotomy, according to the practice of the surgeon. During the last 15 years, almost all thymomas were operated on through a sternotomy, and these patients underwent associated systematic thymectomy.

The prognostic factors studied were Masaoka stage, completeness of resection, histologic classification, association with $\mathrm{MG}$, and use of postoperative radiotherapy.

Statistical analysis. Percentage comparisons were made by the continuity-corrected $\chi^{2}$ test. Survival and disease-free survival were calculated by the actuarial method and compared by the log-rank test. ${ }^{10}$ Multivariate analysis was performed by the Cox proportional hazard model by means of BMDP statistical software. ${ }^{11}$ Results were considered significant if the $p$ value was less than 0.05 .

\section{Results}

Extent of thymoma and surgical procedure. All 307 patients were operated on; sternotomy, anterolateral thoracotomy, and posterolateral thoracotomy were performed in $55 \%, 30 \%$, and $15 \%$ of the cases, respectively. At the time of the surgical procedure, thymoma was noninvasive in $52 \%$ of cases. When invasive, the tumor involved pleura $(78 \%)$, pericardium $(45 \%)$, lung $(30 \%)$, phrenic nerve $(30 \%)$, or superior vena caval system $(27 \%)$. In 19 patients, the thymoma was initially metastatic; all metastases were intrathoracic (pleuropulmonary or pericardial), and all metastatic thymomas were also invasive to neighboring tissues.

Two hundred ninety patients $(94.5 \%)$ underwent tumor resection and $17(5.5 \%)$ underwent biopsy only. Thymoma resection was enlarged to the pleura, pericardium, or both in 139 cases; to the lung in 27 cases ( 22 wedge resections, four pneumonectomies, one lobectomy); to one phrenic nerve in 14 cases; to the superior vena caval system in 20 cases (17 innominate vein resections, three superior vena caval resections with graft reconstructions). Eightysix percent of the patients underwent associated thymectomy. Some patients operated on through a posterolateral approach did not undergo thymectomy. In eight patients, a second small thymoma was identified by systematic histologic analysis of the thymus.
Clinical and histologic staging. According to Masaoka and coworkers, ${ }^{8}$ thymomas are classified as follows: stage I, macroscopically completely encapsulated, and microscopically no capsular invasion; stage IIa, macroscopic invasion into surrounding fatty tissue, or mediastinal pleural tissue; stage $\mathrm{IIb}$, microscopic invasion into capsule; stage III, macroscopic invasion into neighboring organ (pericardium, great vessels, or lung); stage IVa, pleural or pericardial dissemination; stage IVb, lymphogenous or hematogenous metastasis.

One hundred thirty-five thymomas $(44 \%)$ were stage I, $70(23 \%)$ were stage II, $83(27 \%)$ were stage III, and $19(6 \%)$ were stage IV. In addition to this classification, we divided patients with stage I tumors into two subgroups; 26 of these patients had significant peritumoral adherences assessed by intraoperative analysis of the surgeon, although the tumor was macroscopically encapsulated and did not have capsular invasion. These 26 cases were classified as stage $\mathrm{Ib}$; those without peritumoral adherence were classified as stage Ia. Among the 288 nonmetastatic thymomas, according to the surgeon and pathologist's analysis, $248(86 \%)$ underwent complete resection (all stage I or II, $50 \%$ of stage III) and $40(14 \%)$ underwent incomplete resection (28) or biopsy (12). Among the 19 cases of metastatic thymoma, we performed 12 complete resections, two incomplete resections, and five biopsies. The distribution of the patients according to Masaoka clinical staging was similar in the patients with MG and with NAD.

Histologic classification was made according to the work of Verley and Hollmann ${ }^{9}$ as four types: spindle and oval cell thymomas (type 1), lymphocyte-rich thymomas (type 2), differentiated epithelial-rich thymomas (type 3), and undifferentiated epithelial-rich thymomas (type 4). Sixty-seven (22\%) thymomas were type $1,77(25 \%)$ were type 2,139 $(45 \%)$ were type 3 , and $24(8 \%)$ were type 4 . The correlations between Masaoka clinical stage and histologic type are summarized in Table I. Histologic type 3 and 4 tumors were often invasive or metastatic. The mean age of the patients with type 1 thymomas was significantly higher than that of patients with other types $(56 \pm 12$ vs $47 \pm 13$ years, $p<0.01$ ).

Operative morbidity and mortality. Two hundred eighty-one patients $(91.5 \%)$ had uneventful postoperative courses. Five patients $(1.6 \%)$ died of respiratory, cardiac, or embolic complications. Postoperative mortality was not influenced by MG. 
Table I. Masaoka clinical stage according to histologic classification

\begin{tabular}{|c|c|c|c|c|c|c|c|c|}
\hline & \multicolumn{2}{|c|}{ Type $1(n=67)$} & \multicolumn{2}{|c|}{ Type $2(n=77)$} & \multicolumn{2}{|c|}{ Type $3(n=139)$} & \multicolumn{2}{|c|}{ Type $4(n=24)$} \\
\hline & No. & $\%$ & No. & $\%$ & No. & $\%$ & No. & $\%$ \\
\hline Stage I & 52 & 78 & 48 & 62 & 35 & 25 & 0 & 0 \\
\hline Stage II & 7 & 10 & 17 & 22 & 45 & 32 & 1 & 4 \\
\hline Stage III & 7 & 10 & 11 & 14 & 44 & 32 & 21 & 88 \\
\hline Stage IV & 1 & 1 & 1 & 1 & 15 & 11 & 2 & 8 \\
\hline
\end{tabular}

Percentages do not necessarily add to $100 \%$ as a result of rounding.

Table II. Cause of death according to Masaoka clinical stage

\begin{tabular}{lcccc}
\hline & $\begin{array}{c}\text { Stage I } \\
(n=135)\end{array}$ & $\begin{array}{c}\text { Stage II } \\
(n=70)\end{array}$ & $\begin{array}{c}\text { Stage III } \\
(n=83)\end{array}$ & $\begin{array}{c}\text { Stage IV } \\
(n=19)\end{array}$ \\
\hline Total deaths & 29 & 10 & 39 & 14 \\
Postoperative deaths & 3 & $1^{*}$ & 1 & $2^{*}$ \\
Tumor & 1 & 2 & 20 & 9 \\
Autoimmune disease & 7 & 3 & 12 & 1 \\
Miscellaneous disease & 15 & 2 & 6 & 2 \\
Unknown & 3 & 2 & 0 & 0 \\
\hline
\end{tabular}

*Two of these three patients died after another resection for recurrence.

Twenty-one patients $(6.9 \%)$ had complications necessitating medical treatment: pulmonary infections (12), respiratory failure (eight), parietal infections (five), and pulmonary embolism (two). Fifty-eight percent of the 196 patients with MG required postoperative mechanical ventilation for longer than 3 days. The 12 pulmonary infections and the eight respiratory failures occurred in patients with $\mathrm{MG}$.

Adjuvant therapy. Adjuvant therapy was not carried out according to a protocol in this retrospective study. Our policy was to perform postoperative radiotherapy in cases of invasive thymoma. Finally, postoperative radiotherapy was performed in $13 \%$ of cases of stage I tumors, $78 \%$ of stage II cases, and $83 \%$ of stage III cases. Among the 19 metastatic cases of thymoma (stage IV), $90 \%$ of the patients had postoperative radiotherapy, chemotherapy, or both.

Long-term results. Follow-up data were obtained for 299 patients until either death or completion of the study (January 1994). Eight patients were unavailable for follow-up. Mean follow-up was 8 years, and follow-up exceeded 10 years in one-third of the cases (median follow-up 5.5 years, range 1 to 28 years).

At completion of the study, 92 patients had died. Thirty-two $(35 \%)$ died of progressive tumor, 23 $(25 \%)$ died of progressive autoimmune disease, 25 $(27 \%)$ died of miscellaneous diseases, and $7(8 \%)$ of postoperative complications. The cause of death remained unknown in five patients $(5 \%)$, all of whom underwent complete resection of a stage I or II spindle cell or lymphocyte-rich thymoma. Table II shows the cause of death according to Masaoka clinical stage.

The overall actuarial 10 - and 15-year survivals were respectively $67 \%$ and $57 \%$. The 10 -year survivals were $70 \%$ for patients with MG, $74 \%$ for patients with $\mathrm{OAD}$, and $62 \%$ for patients with NAD; there were no significant differences among these three groups of patients. Fig. 1 shows the actuarial survival curves according to Masaoka clinical stage. No significant difference was found between survival curves of patients with stage I and II tumors ( $p=$ 0.70 ). On the other hand, survival was significantly longer for patients with stage II tumors than those with stage III tumors (log rank test $p<0.001$ ). Similarly a borderline significant difference was found between survival curves of patients with stage $\mathrm{IIa}$ and IIb tumors (10-year survivals $100 \%$ and $74 \%$, respectively, $p=0.09$ ). No significant difference was found between survival curves of patients with stage III and stage IV tumors $(p=0.25)$.

Regarding the completeness of thymoma resection, the actuarial 10-year survival for patients with complete resection was significantly higher than those of patients with incomplete resection or biopsy $(76 \%$ and $28 \%$, respectively, $\log$ rank test $p<$ 0.0001; Fig. 2). Furthermore, survival was significantly higher among patients with stage III disease that was completely resected than among those with incomplete resection or biopsy; 10-year survivals were respectively $75 \%$ and $31 \%$, respectively $(\log$ rank test $p<0.001$, confidence intervals $57 \%$ to $91 \%$ and $15 \%$ to $46 \%$, respectively). Survival was also significantly longer for patients with completely resected stage III thymomas than for patients with completely resected stage IV thymomas. Ten-year survivals were $75 \%$ and $42 \%$, respectively $(p<0.05$, confidence intervals $57 \%$ to $91 \%$ and $12 \%$ to $72 \%$, respectively). On the other hand, although survival was higher among patients with completely resected 


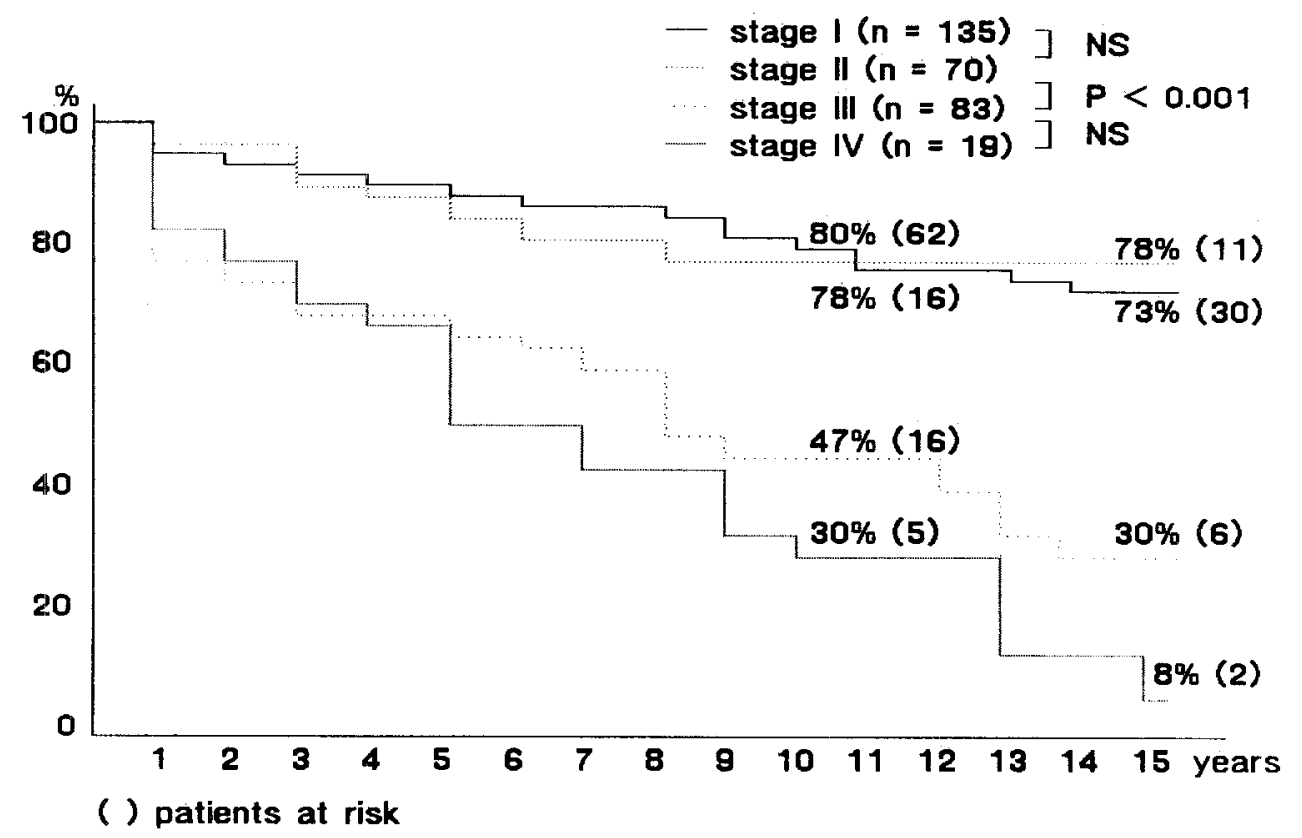

Fig. 1. Actuarial survival according to Masaoka clinical stage. NS, Not significant.

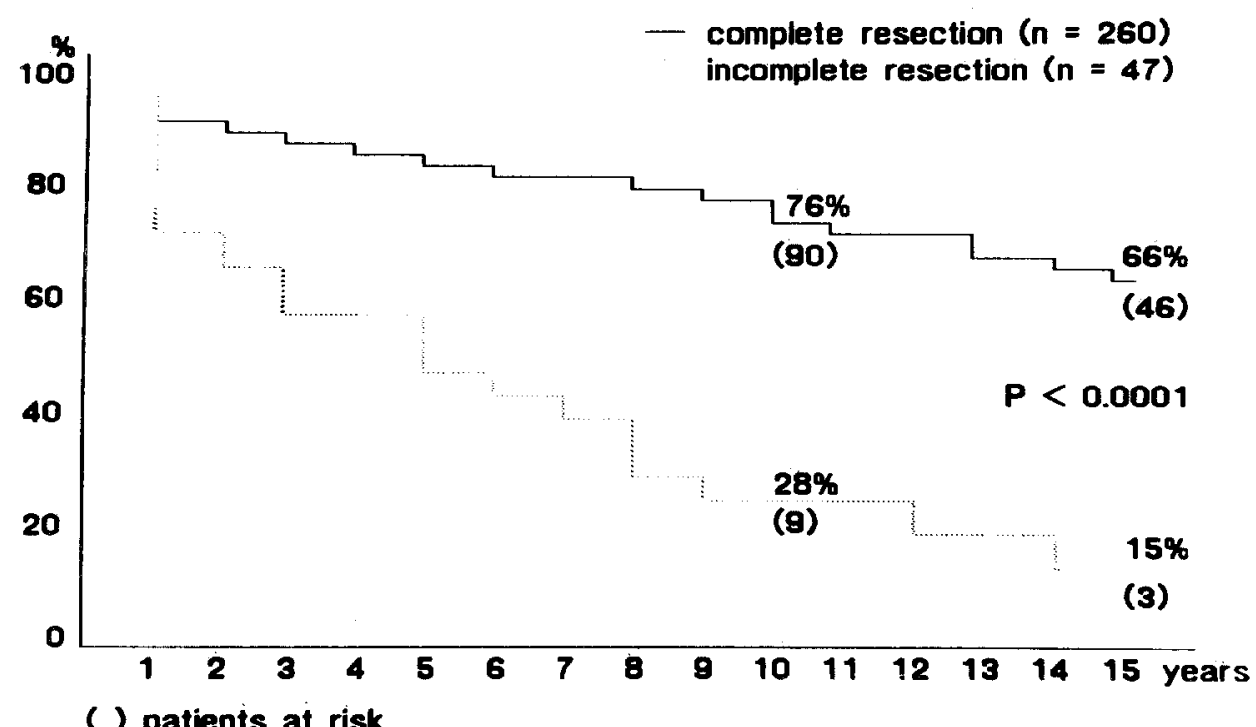

Fig. 2. Actuarial survival according to completeness of resection. NS, Not significant.

stage IV thymomas than among those with incompletely resected stage IV thymomas (5-year survivals $64 \%$ and $34 \%$, respectively, confidence intervals $36 \%$ to $91 \%$ and $0 \%$ to $72 \%$, respectively), the difference was not statistically significant $(p=0.18)$. Among patients with stage III thymomas, survivals were quite similar for patients with incomplete resections and those with biopsies (10-year survivals
$29 \%$ and $35 \%$, respectively, confidence intervals $11 \%$ to $47 \%$ and $5 \%$ to $65 \%$, respectively, $\mathrm{p}=0.85$ ).

Among the 260 cases of completely resected thymoma, 24 patients $(9 \%)$ had recurrence. Disease recurred an average of 5.5 years after operation (median 3.5 years, range 2 to 16 years). Disease recurrence occurred an average of 8.5 years after operation for histologic type 1 or 2 tumors, 5 years 


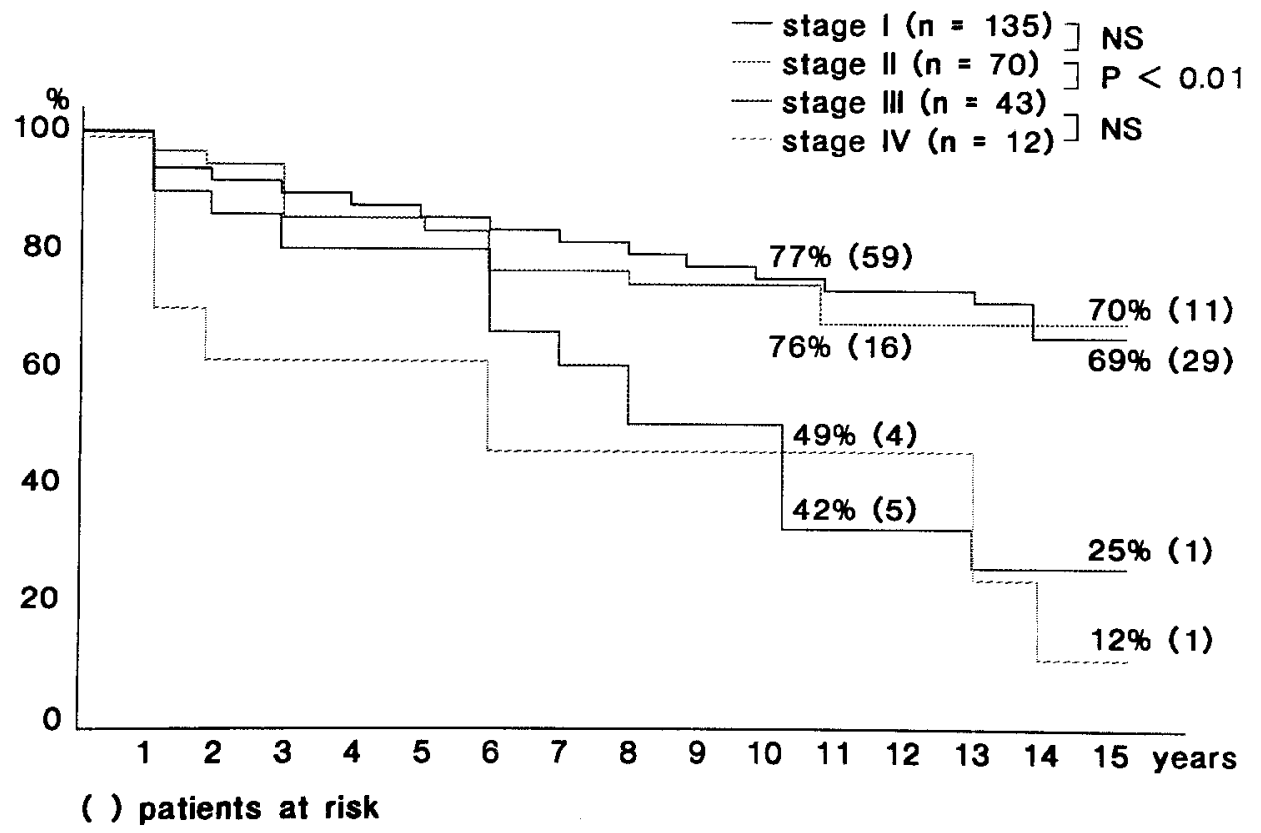

Fig. 3. Disease-free survival according to Masaoka clinical stage in patients with complete resection.

for type 3 , and 3.5 years for type 4 . Recurrence was always in the chest (mediastinal 10, pleural or lung 12, both 2). One patient had a concurrent liver metastasis. Observed recurrence rates were $3.7 \%$ (five patients) among patients with stage I thymomas, $7 \%$ (five patients) among patients with stage II thymomas, $16 \%$ (seven patients) among patients with completely resected stage III thymomas, and $58 \%$ (seven patients) among patients with completely resected stage IV thymomas $(p<0.001)$. Among the 135 patients with stage I disease, the five recurrences occurred in the subgroup of patients with stage $\mathrm{Ib}$ disease (with peritumoral adherence); there were no recurrences observed in patients with stage Ia thymomas. The recurrence rate was significantly higher among patients with stage $\mathrm{Ib}$ disease than among those with stage Ia disease $(p<0.001)$.

To better assess the aggressiveness of thymomas, we also analyzed the disease-free survival curves. Fig. 3 shows the disease-free survival curves for patients with complete resection, according to Masaoka clinical stage. There was a significant difference between disease-free survivals for patients with completely resected stage II and stage III tumors $(p<0.01)$. No significant differences were found between stage I and stage II tumors, nor were there any differences found between completely resected stage III and stage IV tumors ( $p=0.80$ and $p=0.60$, respectively).

Eighteen of the 24 patients with recurrence underwent another resection; 11 patients underwent complete resection and seven underwent incomplete resection. Survival was significantly longer among patients with complete resection than among those with incomplete resection; 10-year survivals were $53 \%$ and $11 \%$, respectively (log rank test $p<0.01$, confidence intervals $19 \%$ to $87 \%$ and $0 \%$ to $32 \%$, respectively). Similarly, five patients with incompletely resected stage III thymomas underwent reoperation after radiotherapy or chemotherapy. Complete resection was performed in two cases; one patient underwent incomplete resection and two underwent exploratory procedures. All these patients died within 3 years, even in cases of complete resection.

In our experience, postoperative radiotherapy was advised in cases of invasive thymoma to decrease recurrence. Among the 114 patients with completely resected invasive thymomas (stage II or stage III), $90(80 \%)$ underwent adjuvant radiotherapy and 24 did not receive any adjuvant treatment. Fig. 4 shows disease-free survival curves for the 90 patients with completely resected invasive thymomas who underwent postoperative radiotherapy and of the 24 pa- 


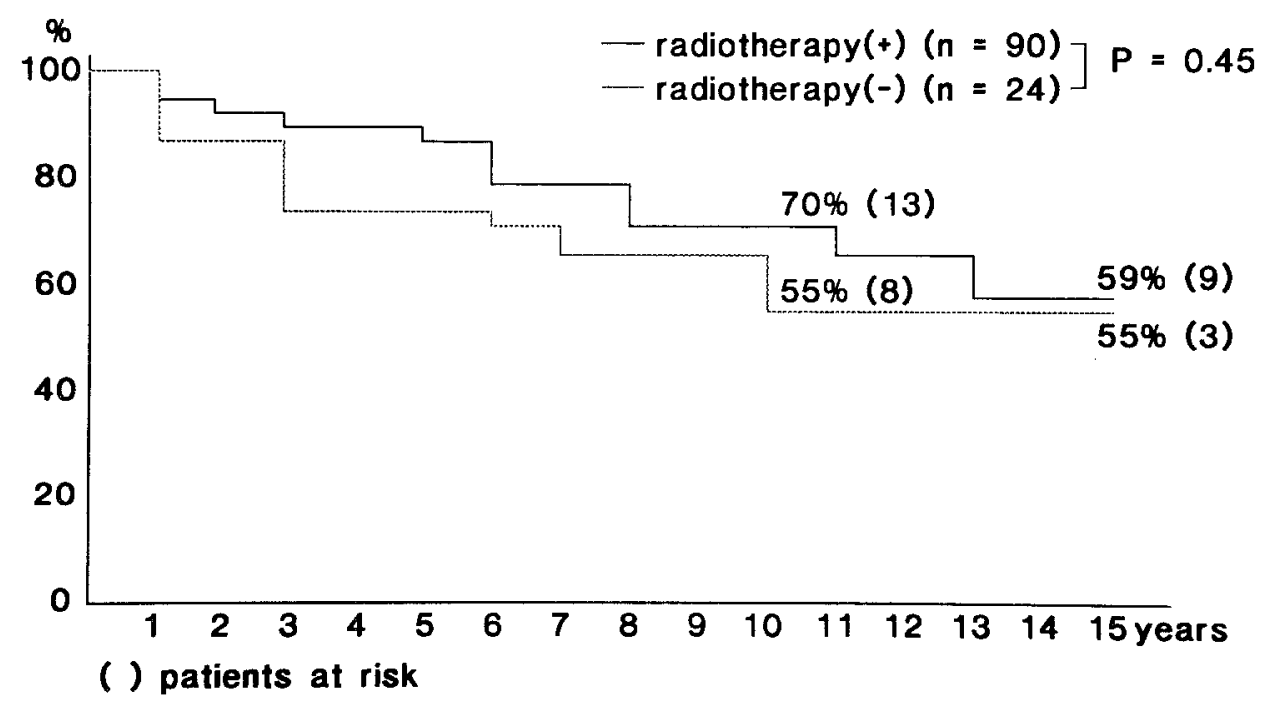

Fig. 4. Disease-free survival according to postoperative radiotherapy in patients with completely resected invasive thymomas.

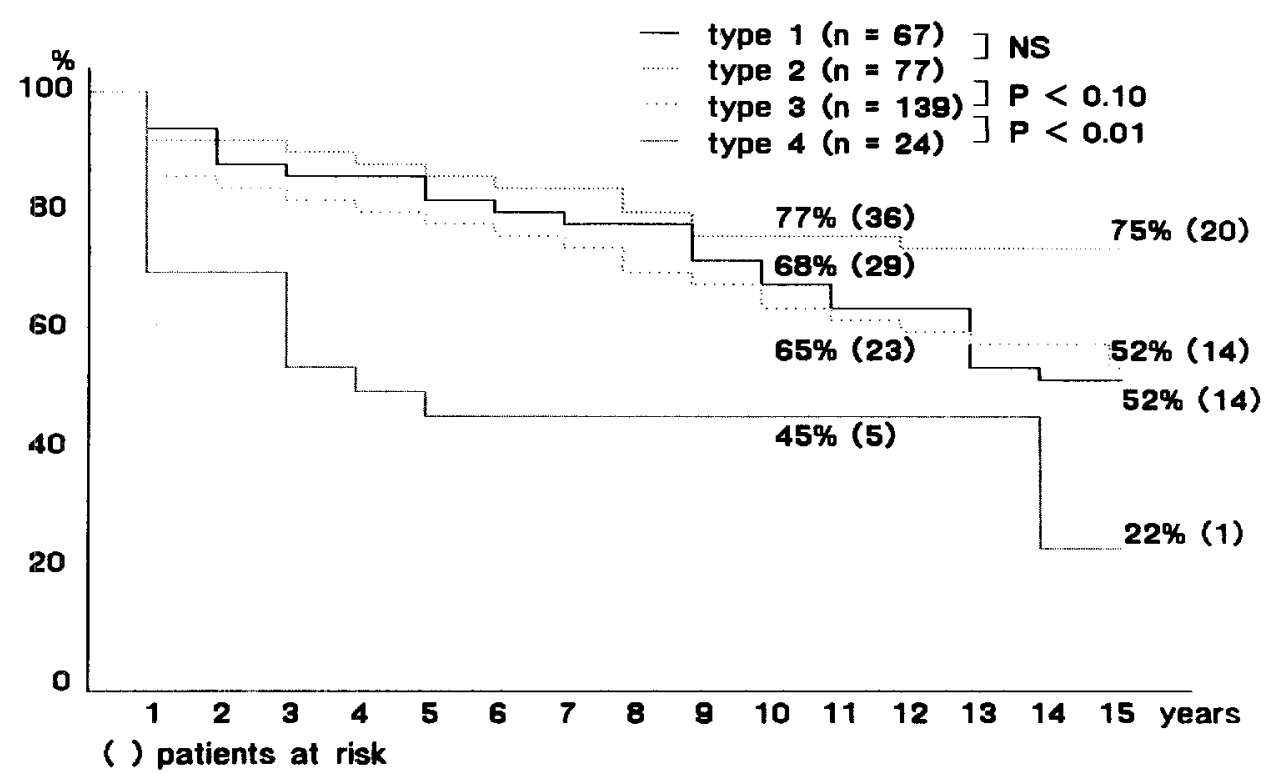

Fig. 5. Actuarial survival according to histologic classification. NS, Not significant.

tients who did not undergo postoperative radiotherapy. No significant differences were found.

Fig. 5 shows the 10 -year survival according to histological classification. No significant difference was found between curves for type 1 and 2 diseases. On the other hand, we found a borderline significant difference between survivals for patients with type 2 and 3 thymonas $(p=0.07)$ and a highly significant difference between patients with type 3 and 4 disease $(p<0.01)$.
Finally, three prognostic factors were established in univariate analysis: completeness of resection, Masaoka clinical stage, and histologic classification. Because histologic classification, Masaoka clinical stage, and completeness of resection all seemed linked, we performed a multivariate analysis. The following covariables were analyzed by multivariate analysis to assess their independent prognostic value for survival: completeness of resection, Masaoka clinical stage, histologic classification, association 
with MG, and postoperative radiotherapy. By this method, the completeness of the resection was found to be the sole significant prognostic factor $(p<0.00001)$. Masaoka clinical stage should no longer appear as a prognostic factor because completeness of resection and Masaoka clinical stage were closely linked. The same criterion applies to the histologic classification. In contrast, when we used a four-covariable model excluding complete resection factor, Masaoka stage was the sole independent prognostic factor $(p<0.00001)$. Histologic classification never appeared as a prognostic factor in multivariate analysis.

\section{Discussion}

Prognostic factors for thymoma are difficult to define because these tumors are uncommon, polymorphic, and slow growing. A large series and long-term follow-up are needed to try to define prognostic factors. Furthermore, $65 \%$ of the causes of death were independent of the tumor's progress (complications of an autoimmune diseases or miscellaneous diseases); survival and the disease-free survival curves for patients with complete resection therefore do not exactly reflect the aggressiveness of the tumor. We therefore decided to also compare recurrence rates.

MG is the autoimmune disease most frequently associated with thymoma, and its incidence is generally $30 \%$ or more in most reported series., 4,7 The unusually high proportion of patients with $\mathrm{MG}$ in our series (64\% of the patients) is explained by a specific recruitment of thymectomy for $\mathrm{MG}^{12}$; some of these patients also had a thymoma and required a wider surgical procedure. The prognostic value of MG is controversial. Maggi and associates ${ }^{4}$ demonstrated a better prognosis for patients with $\mathrm{MG}$, linked to an earlier thymoma diagnosis and less invasive thymoma in this group. Monden and colleagues $^{2}$ found a lower recurrence rate among patients with $\mathrm{MG}$, even in cases with similar clinical stages. In our experience, we found an equal distribution of the clinical stages in patients with $\mathrm{MG}$ and patients with NAD. Furthermore, survivals of patients with $\mathrm{MG}$ and patients with NAD were similar, as in most series. ${ }^{1,3,6,7}$

The clinical staging system described by Masaoka and coworkers ${ }^{8}$ reflects the highly variable malignant potential of these tumors and is considered by most authors as the major prognostic factor. ${ }^{1,3,4,6}$ Clinical staging must be supplemented by histologic research. Wilkins and colleagues ${ }^{6}$ pointed out the risk of missing a microscopic capsular invasion. Similarly, a tumoral invasion must be differentiated from a peritumoral adherence. Surprisingly, this classification does not take into account completeness of resection, which for most malignant tumors is one of the best prognostic indicators. In our series, we also found the overall prognostic significance of Masaoka clinical stage in univariate analysis, with a worse prognosis in cases of invasive or metastatic tumor than in cases of noninvasive tumor. We found, however, a significant difference in survival between patients with completely resected stage III tumors and those who underwent incomplete resection or biopsy only of stage III tumors. Similarly in stage IV thymomas, there was a trend toward better survival for patients with complete resection than for patients with incomplete resection. The absence of significance was probably a result of the lack of power of the test (short samples).

As a result, completeness of resection should be taken into account in a clinical-pathologic classification, as some other authors ${ }^{2,5}$ have pointed out. Our results demonstrated that completeness of resection was the major survival prognostic factor in univariate analysis, and this contention was supported by the multivariate analysis. This prognostic factor was more significant than Masaoka clinical stage. Furthermore, stage I should be divided into two subgroups; in fact, even though the disease-free survival curves were quite similar for patients with stage Ia and $\mathrm{Ib}$ disease, the five recurrences observed in the stage I group all occurred in patients with stage $\mathrm{Ib}$ thymomas. We therefore propose a modified clinical-pathologic staging system as follows: stage Ia, complete resection of a thymoma, macroscopically completely encapsulated and with no microscopic capsular invasion; stage $\mathrm{Ib}$, complete resection of a thymoma, macroscopically completely encapsulated and with no microscopic capsular invasion, but with peritumoral adherences; stage IIa, complete resection of a thymoma with macroscopic invasion into surrounding fatty tissue or mediastinal pleura; stage IIb, microscopic invasion into capsule; stage IIIa, complete resection of a thymoma with macroscopic invasion into neighboring organ (pericardium, great vessels, or lung); stage IIIb, incomplete resection or biopsy of a thymoma with macroscopic invasion into neighboring organ (pericardium, great vessels, or lung); stage IVa, complete resection of a metastatic thymoma; and stage IVb, incomplete resection of a metastatic thymoma.

In cases of invasive thymoma, some authors ${ }^{4}$ have 
found that subtotal resection allows a better prognosis than biopsy alone. As in the series of Nakahara and associates ${ }^{5}$ and Cohen and coworkers, ${ }^{13}$ we could not confirm these results; in our series of patients with stage III thymomas, the prognoses were similar in cases of incomplete resection and biopsy. According to our experience, surgical resection should be performed only in a curative attempt. Neoadjuvant radiotherapy or chemotherapy should be tested when the tumor seems marginally operable. ${ }^{13-17}$ Although survival was not significantly longer among patients with completely resected stage IV thymomas than among those with incomplete resection or biopsy of stage IV thymomas, we recommend surgical resection for these patients if a curative resection seems possible because long-term survivors were observed only in the group with resection, and neoadjuvant radiotherapy or chemotherapy could also be tested for these patients.

Postoperative radiotherapy is usually recommended for invasive thymomas to reduce recurrence. ${ }^{4,6,18}$ Most disease recurred well after the surgical resection, and long-term follow-up is necessary to study this problem. Some authors ${ }^{2,5}$ recommend postoperative radiotherapy for all thymomas; in our experience, no recurrences were observed after resection of stage Ia thymomas; we therefore do not recommend postoperative radiotherapy for this group of patients. For all other patients undergoing resection, we continue to recommend postoperative radiotherapy even though our study did not find any significant difference in terms of diseasefree survival between irradiated patients and nonirradiated patients. This was not a randomized study, and further studies are necessary to draw a definite conclusion.

The value of histologic classification of thymomas is still controversial; various classifications have been used. ${ }^{9,} 19,20$ Some authors do not pay any attention to histologic type. ${ }^{4,5}$ By others, epithelial tumors were seen to have an adverse prognostic factor because of the high frequency of invasive tumors of this histologic type. ${ }^{1,19}$ In univariate analysis, we also found a prognostic value of histologic classification, with a worse prognosis for patients with differentiated epithelium-rich thymomas or undifferentiated epithelium-rich thymomas than for those with lymphocyte-rich or spindle or oval cell thymomas. The histological classification is closely linked to the clinico-pathological staging (Table II), however, and finally to the possibility of performing a complete resection. In multivariate analysis, the histologic classification did not appear as a prognostic factor. In our experience, prognosis could be better evaluated by intraoperative identification and evaluation (extension, possibility of complete resection) than by histologic analysis.

In a recent article, Martinez and associates ${ }^{21}$ found Marino and Müller-Hermelinck's histologic classification $^{20}$ to be an independent prognostic factor. We did not use that histologic classification. Surprisingly, these authors did not observe any recurrence of medullary and mixed thymomas, which they considered benign tumors with no risk of recurrence. We did not agree with their conclusions; our histologic type 1 is strictly comparable, as far as our pathologists are concerned, with the medullary subgroup of the Marino and Müller-Hermelinck classification $^{20}$; in this subgroup of patients, we observed not only some invasive thymomas (seven stage II, seven stage III, and one stage IV among the 67 patients) but also two long-term recurrences, with two deaths related to recurrence. In light of these facts, we could not consider this subgroup of thymomas to be benign tumors. Furthermore in the series of Martinez and associates, ${ }^{21}$ Masaoka clinical stage remained the best prognostic factor, more significant than histologic classification, so we think that our new clinical pathologic staging, including completeness of resection, remains the best prognostic indicator.

Finally, in our experience only patients with stage Ia thymomas had no recurrence, and the prognoses of stage $\mathrm{Ib}$ and II tumors are quite similar. The invasion of neighboring organs (stage III) significantly increases the risk of recurrence even in cases of complete resection (stage IIIa). In stage IIIb, the prognosis was similar in cases with incomplete resection and biopsy alone. Long-term survivors were observed after complete resection of metastatic thymomas (stage IVa). Even though the benefits of adjuvant radiotherapy for invasive thymomas was not demonstrated, radiotherapy is recommended for these patients until further studies have been performed. Neoadjuvant chemotherapy or radiotherapy should be tested in prospective trials for grossly invasive or metastatic thymomas, but surgical resection should be performed whenever complete resection seems possible. Further studies are necessary to evaluate the value of histologic classification. A unified histologic and clinical-pathologic classification is indispensable for the sake of further studies (adjuvant or neoadjuvant treatment, research of other prognostic factors). 
We gratefully acknowledge $\mathrm{F}$. Lacaine, $\mathrm{MD}$, and Y. Flamant, MD, for their help with statistical analysis.

\section{REFERENCES}

1. Pescarmona E, Rendina EA, Venuta F, D'Arcangelo E, Pagani M, Ricci C, et al. Analysis of prognostic factors and clinicopathological staging of thymoma. Ann Thorac Surg 1990;50:534-8.

2. Monden Y, Nakahara K, Iioka S, Nanjo S, Ohno K, Fujii $\mathrm{Y}$, et al. Recurrence of thymoma: clinicopathological features, therapy, and prognosis. Ann Thorac Surg 1985; $39: 165-9$.

3. Shamji F, Pearson FG, Todd TR, Ginsberg RI, Ilves R, Cooper JD. Results of surgical treatment for thymoma. J Thorac Cardiovasc Surg 1984;87:43-7.

4. Maggi G, Casadio C, Cavallo A, Cianci R, Molinatti M, Ruffini E. Thymoma: results of 241 operated cases. Ann Thorac Surg 1991;51:152-6.

5. Nakahara K, Ohno K, Hashimoto J, Maeda H, Miyoshi S, Sakurai M, et al. Thymoma: results with complete resection and adjuvant postoperative irradiation in 141 consecutive patients. J Thorac Cardiovasc Surg 1988;95:1041-7.

6. Wilkins EW, Grillo HC, Scannell JG, Moncure AL, Mathisen DJ. Role of staging in prognosis and management of thymoma. Ann Thorac Surg 1991;51:888-92.

7. Lewis JE, Wick MR, Scheithauer BW, Bernatz PE, Taylor WF. Thymoma: a clinicopathologic review. Cancer 1987;60: 2727-43.

8. Masaoka A, Monden Y, Nakahara K, Tanioka T. Follow-up study of thymoma with special reference to their clinical stages. Cancer 1981;48:2485-92.

9. Verley JM, Hollmann KH. Thymoma: a comparative study of clinical stages, histologic features, and survival in 200 cases. Cancer 1985;55:1074-86.
10. Kaplan EL, Meier P. Non parametric estimation from incomplete observation. J Am Stat Assoc 1958;53:457-81.

11. Dixon WJ. BMDP statistical software. Berkeley, (CA), University of California Press, 1981.

12. Le Brigand $H$. Evolution of the indications and results of treatment of myasthenia by thymectomy. Int Surg 1982;67:9-11.

13. Cohen DJ, Ronningen LD, Graeber GM, Deshong JL, Jaffin J, Burge ZR, et al. Management of patients with malignant thymoma. J Thorac Cardiovasc Surg 1984;86:301-7.

14. Chahinian AP, Bhardwaj S, Meyer RJ, Jaffrey IS, Kirschner PA, Holland JF. Treatment of invasive or metastatic thymoma: report of eleven cases. Cancer 1981;47:1752-61.

15. Loehrer PJ, Bonomi P, Goldman S, Reddy S, Faber LP, Jensik $R$, et al. Remission of invasive thymoma due to chemotherapy: two patients treated with cyclophosphamide, doxorubicin and vincristine. Chest 1985;87:377-80.

16. Macchiarini P, Chella A, Ducci F, Rossi B, Testi C, Bevilacqua $G$, et al. Neoadjuvant chemotherapy, surgery, and postoperative radiation therapy for invasive thymoma. Cancer 1991;68:706-13.

17. Kirschner PA. Reoperation for thymoma: report of 23 cases. Ann Thorac Surg 1990;49:550-5.

18. Haniuda $M$, Morimoto $M$, Nishimura $H$, Kobayashi $O$, Yamanda $T$, Iida F. Adjuvant radiotherapy after complete resection of thymoma. Ann Thorac Surg 1992;54:311-5.

19. Levine GD, Rosai J. Thymic hyperplasia and neoplasia: a review of current concepts. Hum Pathol 1978;9:495-515.

20. Marino M, Müller-Hermelink HK. Thymoma and thymic carcinoma: relation of thymoma epithelial cells to the cortical and medullary differentiation of the thymus. Virchows Arch [A] 1985;407:119-49.

21. Martinez LQ, Wilkins EW, Choi N, Efird J, Hug E, Harris NL. Thymoma: histologic subclassification is an independent prognostic factor. Cancer 1994;74:606-17. 